\title{
Correction to: Patient's age and D-dimer levels predict the prognosis in patients with TAFRO syndrome
}

\author{
Hiroshi Kawabata ${ }^{1}$ (D) Shino Fujimoto ${ }^{1} \cdot$ Tomoyuki Sakai $^{1} \cdot$ Hiroto Yanagisawa $^{1} \cdot$ Toshio Kitawaki $^{2}$. \\ Kenji Nara ${ }^{3} \cdot$ Masao Hagihara ${ }^{4} \cdot$ Hiroshi Yamamoto $^{5} \cdot$ Masakuni Tanimizu $^{6} \cdot$ Chikako Kato $^{7} \cdot$ Tomoki Origuchi $^{8}$. \\ Kazutaka Sunami ${ }^{9} \cdot$ Yoshitaka Sunami $^{10} \cdot$ Taro Masunari $^{11}$. Nobuhiko Nakamura ${ }^{12}$. Masanori Kobayashi ${ }^{13}$. \\ Keiko Yamagami ${ }^{14} \cdot$ Katsuhiro Miura $^{15} \cdot$ Kazue Takai $^{16} \cdot$ Sadao Aoki $^{17} \cdot$ Norifumi Tsukamoto $^{18} \cdot$ Yasufumi Masaki $^{1}$
}

Published online: 1 June 2021

(c) Japanese Society of Hematology 2021

\section{Correction to: International Journal of Hematology https://doi.org/10.1007/s12185-021-03159-x}

In the original publication of the article, the abstract was wrongly published with grammatical errors. The correct abstract is given below:

Objectives: To identify prognostic factors for TAFRO syndrome, a rare inflammatory disorder of unknown etiology characterized by thrombocytopenia, anasarca, fever, reticulin myelofibrosis, renal dysfunction, and organomegaly.

The original article can be found online at https://doi.org/10.1007/ s12185-021-03159-x.

Hiroshi Kawabata

hkawabat@kuhp.kyoto-u.ac.jp

1 Department of Hematology and Immunology,

Kanazawa Medical University, 1-1 Daigaku, Uchinada, Ishikawa-ken 920-0293, Japan

2 Department of Hematology and Oncology, Graduate School of Medicine, Kyoto University, Kyoto, Japan

3 Department of Hematology, Seirei Mikatahara General Hospital, Hamamatsu, Japan

4 Department of Hematology, Eiju General Hospital, Tokyo, Japan

5 First Department of Internal Medicine, Shinshu University School of Medicine, Matsumoto, Japan

6 Department of Internal Medicine, Tottori Municipal Hospital, Tottori, Japan

7 Department of General Internal Medicine, Toyota Kosei Hospital, Toyota, Japan

8 Department of Immunology and Rheumatology, Nagasaki University Graduate School of Biomedical Science, Nagasaki, Japan

9 Department of Hematology, NHO Okayama Medical Center, Okayama, Japan
Methods: Data of patients with TAFRO syndrome were extracted from a Japanese patient registry. Patients were divided into groups according to the clinical and laboratory parameters at initial presentation. Cut-off values for the laboratory parameters were determined using receiver operating characteristic curve analysis and by clinical relevance. Patient survival was analyzed by the Kaplan-Meier method. Univariable analysis was performed using log-rank tests. Multivariable analyses were performed with the logistic regression model and the Cox proportional hazards model.

Results: We extracted the data of 83 patients with TAFRO syndrome from the registry. Univariable analysis

10 Department of Hematology, Juntendo University Shizuoka Hospital, Izunokuni, Japan

11 Department of Infectious Disease, Chugoku Central Hospital, Fukuyama, Japan

12 Department of Hematology, Gifu University Graduate School of Medicine, Gifu, Japan

13 Kassai Medical Clinic, Osaka, Japan

14 Department of Internal Medicine, Osaka City General Hospital, Osaka, Japan

15 Division of Hematology and Rheumatology, Department of Internal Medicine, Nihon University Itabashi Hospital, Tokyo, Japan

16 Department of Hematology, Niigata City General Hospital, Niigata, Japan

17 Department of Pathophysiology, Faculty of Pharmaceutical Sciences, Niigata University of Pharmacy and Applied Life Sciences, Niigata, Japan

18 Oncology Centre, Gunma University Hospital, Maebashi, Japan 
identified several potential prognostic factors. Of these factors, age $\geq 60$ years and D-dimer $\geq 18 \mu \mathrm{g} / \mathrm{dL}$ remained significant predictors of poor overall survival in the multivariable Cox proportional hazards model. Based on these results, we developed a simple prognostic scoring system for TAFRO syndrome (TS-PSS).

Conclusion: Patients in our cohort were stratified into low, intermediate, and high-risk groups by the TS-PSS. This system should be verified with independent patient cohorts in future studies.
The original article has been updated.

Publisher's Note Springer Nature remains neutral with regard to jurisdictional claims in published maps and institutional affiliations. 